\title{
Glutathione-Protected Gold Clusters Revisited: Bridging the Gap between Gold(I)-Thiolate Complexes and Thiolate-Protected Gold Nanocrystals
}

\section{Yuichi Negishi, Katsuyuki Nobusada and Tatsuya Tsukuda*}

Research Center for Molecular-Scale Nanoscience and Department of Theoretical Studies, Institute for Molecular Science, Myodaiji, Okazaki 444-8585, Japan. Department of Photoscience, School of Advanced Sciences, The Graduate University for Advanced Studies, Hayama, Kanagawa 240-0193, Japan.

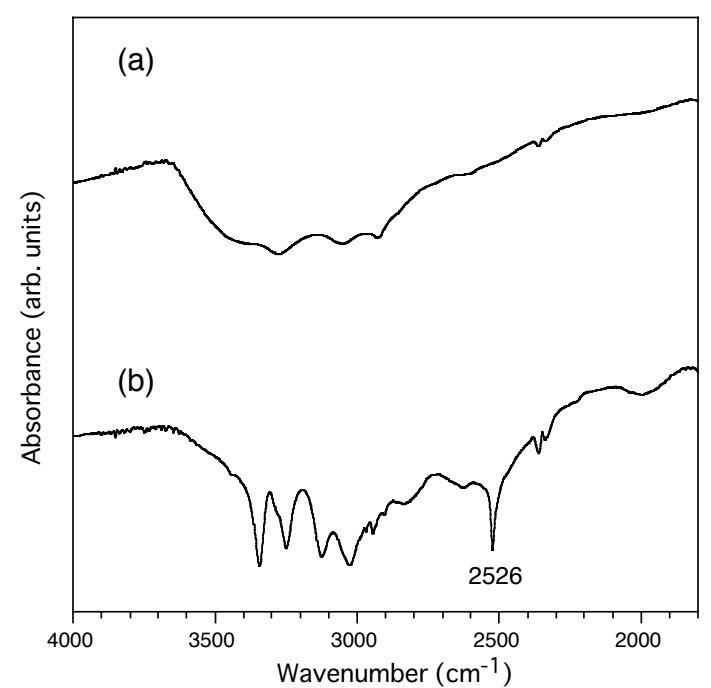

Figure S1. FT-IR spectra of (a) the Au:SG clusters and (b) pure GSH. The peak at $2526 \mathrm{~cm}^{-1}$, which correspond to $\mathrm{S}-\mathrm{H}$ streching vibration mode, disappears in the $\mathrm{Au}: \mathrm{SG}$ clusters.

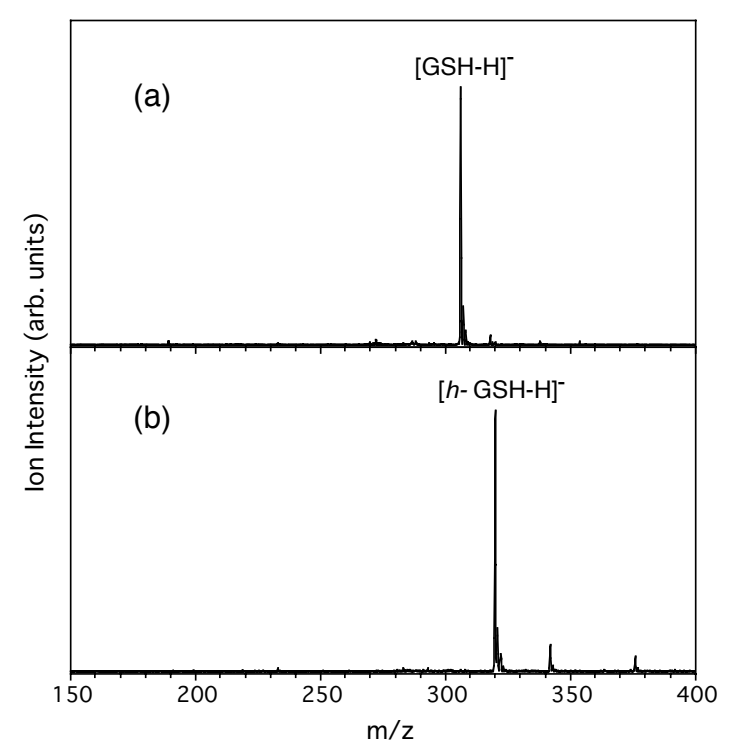

Figure S2. ESI mass spectra of (a)GSH and (b) $h$-GSH.

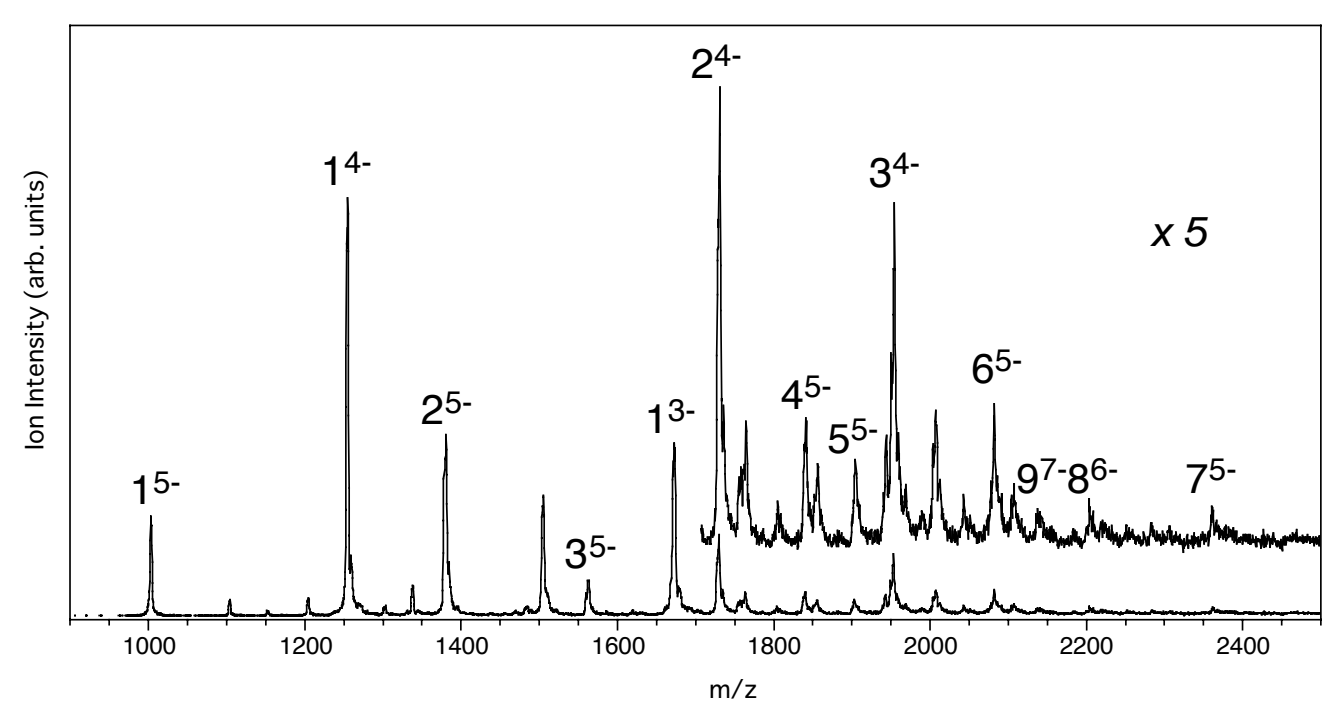

Figure S3. ESI mass spectrum of the as-prepared Au:SG cluster sample. Main peaks are assigned to the compounds listed in Table 1. 


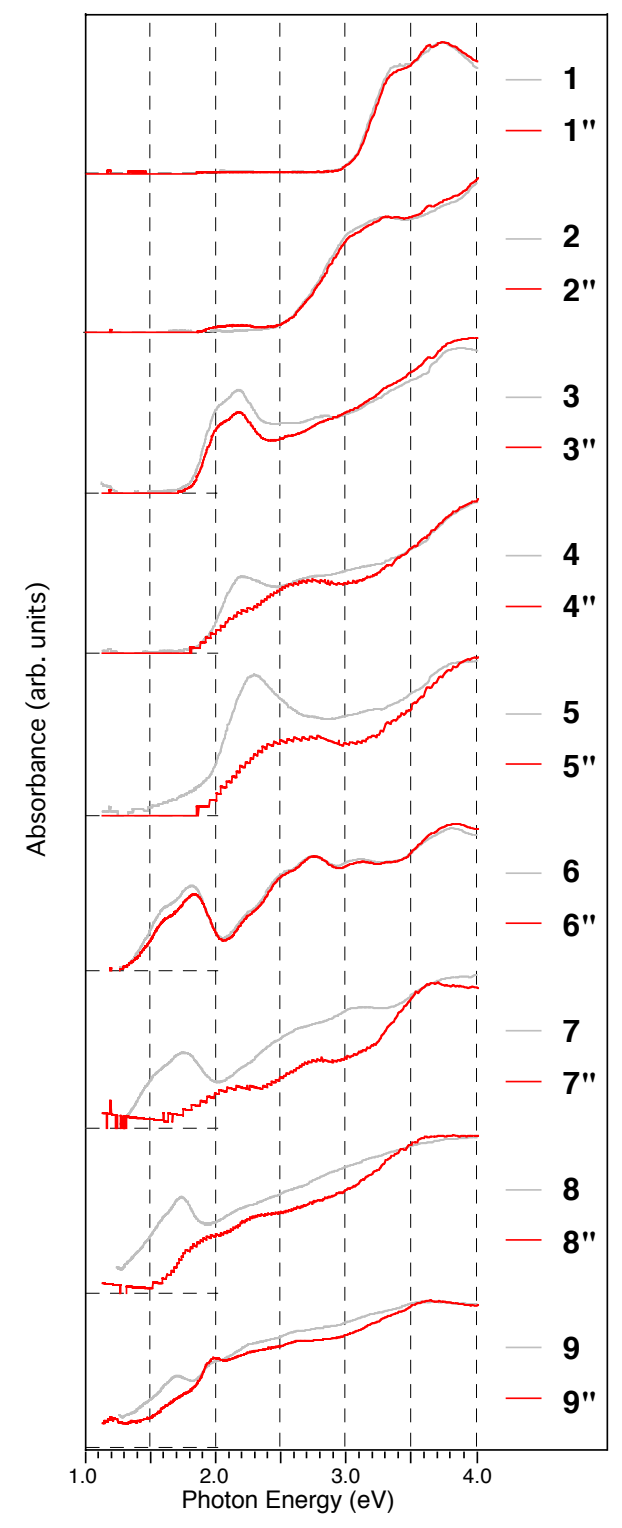

Figure S4. Optical absorption spectra of aqueous solutions of 1-9 ( - ) and 1"-9" ( - ) at ambient temperature.
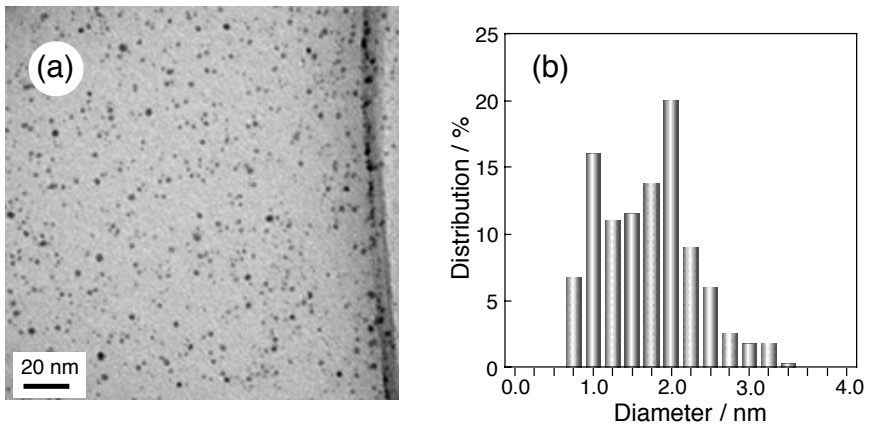

Figure S5. (a) TEM image and (b) size distribution of the Au:SG NCs.

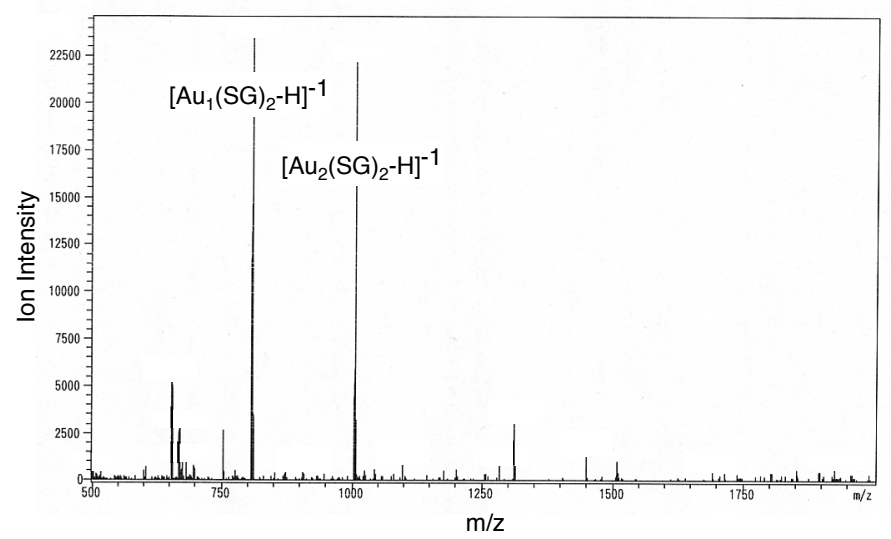

Figure S6. Mass spectrum of 6 recorded by an LCMS-2010A apparatus. 